\title{
Nonlinear integral inequalities on time scales with 'maxima'
}

\author{
Phollakrit Thiramanus ${ }^{1}$, Jessada Tariboon ${ }^{1 *}$ and Sotiris K Ntouyas ${ }^{2}$
}

${ }^{*}$ Correspondence:
jessadat@kmutnb.ac.th
${ }^{1}$ Nonlinear Dynamic Analysis
Research Center, Department of
Mathematics, Faculty of Applied
Science, King Mongkut's University
of Technology North Bangkok,
Bangkok, 10800, Thailand
Full list of author information is
available at the end of the article

available at the end of the article

\begin{abstract}
In this paper, some new types of nonlinear integral inequalities on time scales with 'maxima', which provide explicit bounds on unknown functions, are established. The importance of these integral inequalities is given by their wide applications in qualitative investigations of differential equations with 'maxima'. An example is also presented to illustrate our results.
\end{abstract}

MSC: 34A40; 26D15; 39A13

Keywords: nonlinear integral inequality; time scales; maxima; dynamic equation; differential equations with 'maxima'

\section{Introduction}

The theory of time scales (closed subsets of $\mathbb{R}$ ) was created by Hilger [1] in order to unify continuous and discrete analysis and in order to extend those theories to other kinds of the so-called dynamic equations. Many authors have expounded on various aspects of the theory of dynamic equations on time scales. We refer the reader to the monograph [2] and the references cited therein. Also, a few papers studied the theory of dynamic inequalities on time scales; see, for example, [3-17].

Differential equations with 'maxima' are a special type of differential equations that contain the maximum of the unknown function over a previous interval. Several integral inequalities have been established in the case when maxima of the unknown scalar function is involved in the integral; see [18-21] and the references cited therein.

Recently in [22] we initiated the study of integral inequalities on time scales with 'maxima', where some new integral inequalities were established. The significance of our work in [22] lies in the fact that 'maxima' are taken on intervals $[\beta t, t]$ which have non-constant length, where $0<\beta<1$. Most papers take the 'maxima' on $[t-h, t]$, where $h>0$ is a given constant.

In this paper we continue the study of [22] and investigate some nonlinear dynamic integral inequalities on time scales with 'maxima'. This paper is organized as follows. In Section 2 we give some preliminary results with respect to the calculus on time scales. In Section 3 we deal with our nonlinear dynamic inequalities on time scales with 'maxima'. In Section 4 we give an example to illustrate our main results.

\section{Preliminaries}

In this section, we list the following well-known definitions and some lemmas which can be found in [2] and the references therein.

@ 2014 Thiramanus et al.; licensee Springer. This is an Open Access article distributed under the terms of the Creative Commons Attribution License (http://creativecommons.org/licenses/by/2.0), which permits unrestricted use, distribution, and reproduction in any medium, provided the original work is properly cited. 
Definition 2.1 A time scale $\mathbb{T}$ is an arbitrary nonempty closed subset of the real set $\mathbb{R}$ with the topology and ordering inherited from $\mathbb{R}$.

The forward and backward jump operators $\sigma, \rho: \mathbb{T} \rightarrow \mathbb{T}$ and the graininess $\mu: \mathbb{T} \rightarrow \mathbb{R}_{+}$ are defined, respectively, by

$$
\sigma(t):=\inf \{s \in \mathbb{T} \mid s>t\}, \quad \rho(t):=\sup \{s \in \mathbb{T} \mid s<t\}, \quad \mu(t):=\sigma(t)-t
$$

for all $t \in \mathbb{T}$. If $\sigma(t)>t, t$ is said to be right scattered, and if $\rho(t)<t, t$ is said to be left scattered; if $\sigma(t)=t, t$ is said to be right dense, and if $\rho(t)=t, t$ is said to be left dense. If $\mathbb{T}$ has a right-scattered minimum $m$, define $\mathbb{T}_{k}=\mathbb{T}-\{m\}$; otherwise set $\mathbb{T}_{k}=\mathbb{T}$. If $\mathbb{T}$ has a left-scattered maximum $M$, define $\mathbb{T}^{k}=\mathbb{T}-\{M\}$; otherwise set $\mathbb{T}^{k}=\mathbb{T}$.

Definition 2.2 A function $f: \mathbb{T} \rightarrow \mathbb{R}$ is rd-continuous (rd-continuous is short for rightdense continuous) provided it is continuous at each right-dense point in $\mathbb{T}$ and has a leftsided limit at each left-dense point in $\mathbb{T}$. The set of rd-continuous functions $f: \mathbb{T} \rightarrow \mathbb{R}$ will be denoted by $C_{\mathrm{rd}}(\mathbb{T})=C_{\mathrm{rd}}(\mathbb{T}, \mathbb{R})$.

Definition 2.3 For $f: \mathbb{T} \rightarrow \mathbb{R}$ and $t \in \mathbb{T}^{k}$, the delta derivative of $f$ at the point $t$ is defined to be the number $f^{\Delta}(t)$ (provided it exists) with the property that for each $\varepsilon>0$, there is a neighborhood $U$ of $t$ such that

$$
\left|f(\sigma(t))-f(s)-f^{\triangleright}(t)(\sigma(t)-s)\right| \leq \varepsilon|\sigma(t)-s|
$$

for all $s \in U$.

Definition 2.4 For a function $f: \mathbb{T} \rightarrow \mathbb{R}$ (the range $\mathbb{R}$ of $f$ may be actually replaced by a Banach space), the (delta) derivative is defined at point $t$ by

$$
f^{\triangle}(t)=\frac{f(\sigma(t))-f(t)}{\sigma(t)-t},
$$

if $f$ is continuous at $t$ and $t$ is right scattered. If $t$ is not right scattered, then the derivative is defined by

$$
f^{\Delta}(t)=\lim _{s \rightarrow t} \frac{f(\sigma(t))-f(s)}{\sigma(t)-s}=\lim _{s \rightarrow t} \frac{f(t)-f(s)}{t-s},
$$

provided this limit exists.

Definition 2.5 If $F^{\triangle}(t)=f(t)$, then we define the delta integral by

$$
\int_{a}^{t} f(s) \Delta s=F(t)-F(a)
$$

Lemma 2.1 ([2]) Assume that $v: \mathbb{T} \rightarrow \mathbb{R}$ is strictly increasing and $\widetilde{\mathbb{T}}:=v(\mathbb{T})$ is a time scale. Iff $: \mathbb{T} \rightarrow \mathbb{R}$ is an $r d$-continuous function and $v$ is differentiable with $r$-continuous derivative, then for $a, b \in \mathbb{T}$,

$$
\int_{a}^{b} f(t) v^{\Delta}(t) \Delta t=\int_{v(a)}^{v(b)}\left(f \circ v^{-1}\right)(s) \widetilde{\Delta} s .
$$


Lemma 2.2 ([23]) Assume that $a \geq 0, p \geq q \geq 0$, and $p \neq 0$. Then

$$
a^{\frac{q}{p}} \leq\left(\frac{q}{p} k^{\frac{q-p}{p}} a+\frac{p-q}{p} k^{\frac{q}{p}}\right) \text { for any } k>0
$$

\section{Main results}

For convenience of notation, we let throughout $t_{0} \in \mathbb{T}, t_{0} \geq 0, \mathbb{T}_{0}=\left[t_{0}, \infty\right) \cap \mathbb{T}$ and an interval $[\gamma, \eta]_{\mathbb{T}}=[\gamma, \eta] \cap \mathbb{T}$. In addition, for a strictly increasing function $\alpha: \mathbb{T} \rightarrow \mathbb{R}, \widetilde{\mathbb{T}}=\alpha(\mathbb{T})$ is a time scale such that $\widetilde{\mathbb{T}} \subseteq \mathbb{T}$. For $f \in C_{\mathrm{rd}}(\mathbb{T}, \mathbb{R})$, we define a notation of the composition of two functions on time scales by

$$
f(\gamma) \circ \alpha^{-1}(s)=f\left(\alpha^{-1}(s)\right), \quad \gamma \in \mathbb{T}, s \in \widetilde{\mathbb{T}} .
$$

Example 3.1 Let $f(t)=5^{t^{2}}$ for $t \in \mathbb{T}: \mathbb{N}_{0}^{\frac{1}{2}}=\left\{\sqrt{n}: n \in \mathbb{N}_{0}\right\}$ and $\alpha(t)=t^{2}$ for $t \in \mathbb{T}$. Then we have $\alpha^{-1}(t)=\sqrt{t}$ for $t \in \widetilde{\mathbb{T}}=\mathbb{N}_{0}$ and

$$
f(\gamma) \circ \alpha^{-1}(s)=\left(5^{\gamma^{2}}\right) \circ \sqrt{s}=5^{s}, \quad s \in \widetilde{\mathbb{T}} .
$$

Theorem 3.1 Let the following conditions be satisfied:

(i) The function $\alpha \in C_{\mathrm{rd}}\left(\mathbb{T}_{0}, \mathbb{R}_{+}\right)$is strictly increasing.

(ii) The functions $a, b, p$ and $q \in C_{\mathrm{rd}}\left(\mathbb{T}_{0}, \mathbb{R}_{+}\right)$.

(iii) The function $\phi \in C_{\mathrm{rd}}\left(\left[\beta \tau, t_{0}\right]_{\mathbb{T}}, \mathbb{R}_{+}\right)$, where $0<\beta<1$ and $\tau=\min \left\{t_{0}, \alpha\left(t_{0}\right)\right\}$.

(iv) The function $h \in C\left(\mathbb{R}_{+},(0, \infty)\right)$ is increasing.

(v) The function $u \in C_{\mathrm{rd}}\left([\beta \tau, \infty)_{\mathbb{T}}, \mathbb{R}_{+}\right)$and satisfies the inequalities

$$
\begin{aligned}
u(t) \leq & k+\int_{t_{0}}^{t}\left[p(s) h(u(s))+q(s) h\left(\max _{\xi \in[\beta s, s]_{\mathbb{T}}} u(\xi)\right)\right] \Delta s \\
& +\int_{\alpha\left(t_{0}\right)}^{\alpha(t)}\left[a(\gamma) h(u(\gamma))+b(\gamma) h\left(\max _{\xi \in[\beta \gamma, \gamma]_{\mathbb{T}}} u(\xi)\right)\right] \\
& \circ \alpha^{-1}(s) \widetilde{\Delta} s, \quad t \in \mathbb{T}_{0}, \\
u(t) \leq & \phi(t), \quad t \in\left[\beta \tau, t_{0}\right]_{\mathbb{T}},
\end{aligned}
$$

where $k \geq 0$.

Then, for all $t \in \mathbb{T}_{0}$ satisfying

$$
H(M)+\int_{t_{0}}^{t}\left[p(s)+q(s)+a(s) \alpha^{\triangle}(s)+b(s) \alpha^{\Delta}(s)\right] \Delta s \in \operatorname{Dom}\left(H^{-1}\right),
$$

we have

$$
u(t) \leq H^{-1}\left(H(M)+\int_{t_{0}}^{t}\left[p(s)+q(s)+a(s) \alpha^{\Delta}(s)+b(s) \alpha^{\Delta}(s)\right] \Delta s\right),
$$

where

$$
M=\max \left\{k, \max _{s \in\left[\beta \tau, t_{0}\right]_{\mathbb{T}}} \phi(s)\right\}
$$


and

$$
H(x)=\int_{x_{0}}^{x} \frac{1}{h(r)} d r, \quad x_{0}>0,
$$

which $H(\infty)=\infty$, and $H^{-1}$ is the inverse of $H$.

Proof We define a function $z:[\beta \tau, \infty)_{\mathbb{T}} \rightarrow \mathbb{R}_{+}$by

$$
z(t)= \begin{cases}M+\int_{t_{0}}^{t}\left[p(s) h(u(s))+q(s) h\left(\max _{\xi \in[\beta s, s]} u(\xi)\right)\right] \Delta s & \\ \quad+\int_{\alpha\left(t_{0}\right)}^{\alpha(t)}\left[a(\gamma) h(u(\gamma))+b(\gamma) h\left(\max _{\xi \in[\beta \gamma, \gamma]_{\mathbb{T}}} u(\xi)\right)\right] \circ \alpha^{-1}(s) \widetilde{\Delta} s, & t \in \mathbb{T}_{0}, \\ M, & t \in\left[\beta \tau, t_{0}\right]_{\mathbb{T}},\end{cases}
$$

where $M$ is defined by (3.4). Note that the function $z(t)$ is nondecreasing.

It follows that the inequality

$$
u(t) \leq z(t), \quad t \in[\beta \tau, \infty)_{\mathbb{T}}
$$

holds. Therefore, for $t \in \mathbb{T}_{0}$ and $s \in\left[t_{0}, t\right]_{\mathbb{T}}$, we have

$$
\max _{\xi \in[\beta s, s]_{\mathbb{T}}} u(\xi) \leq \max _{\xi \in[\beta s, s]_{\mathbb{T}}} z(\xi)=z(s)
$$

For $t \in \mathbb{T}_{0}$ and $s \in\left[\alpha\left(t_{0}\right), \alpha(t)\right]_{\mathbb{T}}$, we have

$$
\begin{aligned}
h\left(\max _{\xi \in[\beta \gamma, \gamma]_{\mathbb{T}}} u(\xi)\right) \circ \alpha^{-1}(s) & \leq h\left(\max _{\xi \in[\beta \gamma, \gamma]_{\mathbb{T}}} z(\xi)\right) \circ \alpha^{-1}(s) \\
& =h\left(\max _{\xi \in\left[\beta \alpha^{-1}(s), \alpha^{-1}(s)\right]_{\mathbb{T}}} z(\xi)\right) \\
& =h\left(z\left(\alpha^{-1}(s)\right)\right) \\
& =h(z(\gamma)) \circ \alpha^{-1}(s) .
\end{aligned}
$$

Then, from the definition of $z(t)$ and the above analysis, we get for $t \in \mathbb{T}_{0}$ that

$$
\begin{aligned}
z(t) \leq & M+\int_{t_{0}}^{t}\left[p(s) h(z(s))+q(s) h\left(\max _{\xi \in[\beta s, s]_{\mathbb{T}}} z(\xi)\right)\right] \Delta s \\
& +\int_{\alpha\left(t_{0}\right)}^{\alpha(t)}\left[a(\gamma) h(z(\gamma))+b(\gamma) h\left(\max _{\xi \in[\beta \gamma, \gamma]_{\mathbb{T}}} z(\xi)\right)\right] \circ \alpha^{-1}(s) \widetilde{\Delta} s \\
\leq & M+\int_{t_{0}}^{t}[p(s) h(z(s))+q(s) h(z(s))] \Delta s \\
& +\int_{\alpha\left(t_{0}\right)}^{\alpha(t)}[a(\gamma) h(z(\gamma))+b(\gamma) h(z(\gamma))] \circ \alpha^{-1}(s) \widetilde{\Delta} s \\
= & M+\int_{t_{0}}^{t}[p(s) h(z(s))+q(s) h(z(s))] \Delta s \\
& +\int_{t_{0}}^{t}[a(s) h(z(s))+b(s) h(z(s))] \alpha^{\triangle}(s) \Delta s \\
= & M+\int_{t_{0}}^{t}\left[p(s)+q(s)+a(s) \alpha^{\triangle}(s)+b(s) \alpha^{\triangle}(s)\right] h(z(s)) \Delta s .
\end{aligned}
$$


From inequality (3.6) we have

$$
z^{\triangleright}(t) \leq\left[p(t)+q(t)+a(t) \alpha^{\triangle}(t)+b(t) \alpha^{\triangle}(t)\right] h(z(t))
$$

which implies

$$
\frac{z^{\triangle}(t)}{h(z(t))} \leq p(t)+q(t)+a(t) \alpha^{\triangle}(t)+b(t) \alpha^{\triangle}(t) .
$$

On the other hand, for $t \in \mathbb{T}_{0}$, if $\sigma(t)>t$, then

$$
\begin{aligned}
{[H(z(t))]^{\triangle} } & =\frac{H(z(\sigma(t)))-H(z(t))}{\sigma(t)-t}=\frac{1}{\sigma(t)-t} \int_{z(t)}^{z(\sigma(t))} \frac{1}{h(r)} d r \\
& \leq \frac{z(\sigma(t))-z(t)}{\sigma(t)-t} \frac{1}{h(z(t))}=\frac{z^{\triangle}(t)}{h(z(t))}
\end{aligned}
$$

If $\sigma(t)=t$, then

$$
\begin{aligned}
{[H(z(t))]^{\triangle} } & =\lim _{s \rightarrow t} \frac{H(z(t))-H(z(s))}{t-s}=\lim _{s \rightarrow t} \frac{1}{t-s} \int_{z(s)}^{z(t)} \frac{1}{h(r)} d s \\
& =\lim _{s \rightarrow t} \frac{z(t)-z(s)}{t-s} \frac{1}{h(\omega)}=\frac{z^{\triangle}(t)}{h(z(t))}
\end{aligned}
$$

where $\omega$ lies between $z(s)$ and $z(t)$. Hence from (3.8) and (3.9) we have

$$
[H(z(t))]^{\triangle} \leq \frac{z^{\triangle}(t)}{h(z(t))}
$$

Combining (3.7) and (3.10), we get

$$
[H(z(t))]^{\triangle} \leq p(t)+q(t)+a(t) \alpha^{\triangle}(t)+b(t) \alpha^{\triangle}(t)
$$

An integration for the above inequality with respect to $t$ from $t_{0}$ to $t$ yields

$$
H(z(t)) \leq H(M)+\int_{t_{0}}^{t}\left[p(s)+q(s)+a(s) \alpha^{\triangle}(s)+b(s) \alpha^{\triangle}(s)\right] \Delta s .
$$

Since $H^{-1}$ is an increasing function, we obtain

$$
z(t) \leq H^{-1}\left(H(M)+\int_{t_{0}}^{t}\left[p(s)+q(s)+a(s) \alpha^{\Delta}(s)+b(s) \alpha^{\Delta}(s)\right] \Delta s\right), \quad t \in \mathbb{T}_{0},
$$

which results in (3.3). This completes the proof.

We introduce the following classes of functions in connection with the nonlinearity of the considered integral inequality.

Definition 3.1 ([24]) We will say that a function $h \in C\left(\mathbb{R}_{+}, \mathbb{R}_{+}\right)$is from class $\Phi$ if the following conditions are satisfied: 
(i) $h$ is a nondecreasing function;

(ii) $h(x)>0$ for $x>0$;

(iii) $h(t x) \geq \operatorname{th}(x)$ for $0 \leq t \leq 1, x \geq 0$;

(iv) $\int_{1}^{\infty} \frac{d x}{h(x)}=\infty$.

Definition 3.2 ([24]) We will say that a function $h \in C\left(\mathbb{R}_{+}, \mathbb{R}_{+}\right)$is from class $\Omega$ if the following conditions are satisfied:

(i) $h$ is a nondecreasing function;

(ii) $h(x)>0$ for $x>0$;

(iii) $h(t x) \geq \operatorname{th}(x)$ for $0 \leq t \leq 1, x \geq 0$;

(iv) $h(x+y) \leq h(x)+h(y)$ for $x, y \geq 0$;

(v) $\int_{1}^{\infty} \frac{d x}{h(x)}=\infty$.

Note that the functions $h(x)=\sqrt{x}$ and $h(x)=x$ are from class $\Omega$.

In the case when in place of the constant $k$ involved in Theorem 3.1 we have a function $k(t)$, we obtain the following result using functions from class $\Phi$.

Theorem 3.2 Let the following conditions be satisfied:

(i) The conditions (i)-(iii) of Theorem 3.1 are satisfied.

(ii) The function $h \in C\left(\mathbb{R}_{+}, \mathbb{R}_{+}\right)$and $h \in \Phi$.

(iii) The function $k \in C_{\mathrm{rd}}\left(\mathbb{T}_{0},[1, \infty)\right)$ is nondecreasing.

(iv) The function $u \in C_{\mathrm{rd}}\left([\beta \tau, \infty)_{\mathbb{T}}, \mathbb{R}_{+}\right)$and satisfies the inequalities

$$
\begin{aligned}
u(t) \leq & k(t)+\int_{t_{0}}^{t}\left[p(s) h(u(s))+q(s) h\left(\max _{\xi \in[\beta s, s]_{\mathbb{T}}} u(\xi)\right)\right] \Delta s \\
& +\int_{\alpha\left(t_{0}\right)}^{\alpha(t)}\left[a(\gamma) h(u(\gamma))+b(\gamma) h\left(\max _{\xi \in[\beta \gamma, \gamma]_{\mathbb{T}}} u(\xi)\right)\right] \\
& \circ \alpha^{-1}(s) \widetilde{\Delta} s, \quad t \in \mathbb{T}_{0}, \\
u(t) \leq & \phi(t), \quad t \in\left[\beta \tau, t_{0}\right]_{\mathbb{T}} .
\end{aligned}
$$

Then, for all $t \in \mathbb{T}_{0}$ satisfying

$$
H(N)+\int_{t_{0}}^{t}\left[p(s)+q(s)+a(s) \alpha^{\Delta}(s)+b(s) \alpha^{\triangle}(s)\right] \Delta s \in \operatorname{Dom}\left(H^{-1}\right),
$$

we have

$$
u(t) \leq k(t) H^{-1}\left(H(N)+\int_{t_{0}}^{t}\left[p(s)+q(s)+a(s) \alpha^{\Delta}(s)+b(s) \alpha^{\Delta}(s)\right] \Delta s\right),
$$

where

$$
N=\max \left\{1, \max _{s \in\left[\beta \tau, t_{0}\right]_{\mathbb{T}}} \frac{\phi(s)}{k\left(t_{0}\right)}\right\},
$$

and $H(x)$ is defined by (3.5). 
Proof From inequality (3.11) we obtain for $t \in \mathbb{T}_{0}$

$$
\begin{aligned}
\frac{u(t)}{k(t)} \leq & +\int_{t_{0}}^{t}\left[p(s) \frac{h(u(s))}{k(t)}+q(s) \frac{h\left(\max _{\xi \in[\beta s, s]_{\mathbb{T}}} u(\xi)\right)}{k(t)}\right] \Delta s \\
& +\int_{\alpha\left(t_{0}\right)}^{\alpha(t)}\left[a(\gamma) \frac{h(u(\gamma))}{k(t)}+b(\gamma) \frac{h\left(\max _{\xi \in[\beta \gamma, \gamma]_{\mathbb{T}}} u(\xi)\right)}{k(t)}\right] \circ \alpha^{-1}(s) \widetilde{\Delta} s .
\end{aligned}
$$

Let us define functions $k^{*}:[\beta \tau, \infty)_{\mathbb{T}} \rightarrow \mathbb{R}_{+}$and $w:[\beta \tau, \infty)_{\mathbb{T}} \rightarrow \mathbb{R}_{+}$by

$$
\begin{aligned}
& k^{*}(t)= \begin{cases}k(t), & t \in \mathbb{T}_{0}, \\
k\left(t_{0}\right), & t \in\left[\beta \tau, t_{0}\right]_{\mathbb{T}},\end{cases} \\
& w(t)=\frac{u(t)}{k^{*}(t)}, \quad t \in[\beta \tau, \infty)_{\mathbb{T}} .
\end{aligned}
$$

Note that the function $k^{*}(t)$ is nondecreasing on $t \in[\beta \tau, \infty)_{\mathbb{T}}$.

By conditions (ii) and (iii) of Theorem 3.2, it follows that $\frac{h(u(s))}{k(t)} \leq h\left(\frac{u(s)}{k(t)}\right)$ for $t \in \mathbb{T}_{0}$ and $s \in\left[t_{0}, t\right]_{\mathbb{T}}$. From the monotonicity of $k(t)$ and $\alpha(t)$, we get for $t \in \mathbb{T}_{0}$ and $s \in\left[t_{0}, t\right]_{\mathbb{T}}$ that

$$
\begin{aligned}
\frac{\max _{\xi \in[\beta s, s]_{\mathbb{T}}} u(\xi)}{k(t)} & \leq \frac{\max _{\xi \in[\beta s, s]_{\mathbb{T}}} u(\xi)}{k^{*}(s)} \\
& =\max _{\xi \in[\beta s, s]_{\mathbb{T}}} \frac{u(\xi)}{k^{*}(s)} \leq \max _{\xi \in[\beta s, s]_{\mathbb{T}}} \frac{u(\xi)}{k^{*}(\xi)}
\end{aligned}
$$

For $t \in \mathbb{T}_{0}$ and $s \in\left[\alpha\left(t_{0}\right), \alpha(t)\right] \widetilde{\mathbb{T}}$, we have

$$
\begin{aligned}
\frac{\max _{\xi \in[\beta \gamma, \gamma]_{\mathbb{T}}} u(\xi) \circ \alpha^{-1}(s)}{k(t)} & =\frac{\max _{\xi \in\left[\beta \alpha^{-1}(s), \alpha^{-1}(s)\right]_{\mathbb{T}}} u(\xi)}{k(t)} \\
& \leq \frac{\max _{\xi \in\left[\beta \alpha^{-1}(s), \alpha^{-1}(s)\right]_{\mathbb{T}}} u(\xi)}{k^{*}\left(\alpha^{-1}(s)\right)} \\
& =\max _{\xi \in\left[\beta \alpha^{-1}(s), \alpha^{-1}(s)\right]_{\mathbb{T}}} \frac{u(\xi)}{k^{*}\left(\alpha^{-1}(s)\right)} \\
& \leq \max _{\xi \in\left[\beta \alpha^{-1}(s), \alpha^{-1}(s)\right]_{\mathbb{T}}} \frac{u(\xi)}{k^{*}(\xi)} \\
& =\max _{\xi \in[\beta \gamma, \gamma]_{\mathbb{T}}} \frac{u(\xi)}{k^{*}(\xi)} \circ \alpha^{-1}(s) .
\end{aligned}
$$

From inequalities (3.15), (3.16) and (3.17) and the definition of $w(t)$, we have

$$
\begin{aligned}
w(t) \leq & +\int_{t_{0}}^{t}\left[p(s) h(w(s))+q(s) h\left(\max _{\xi \in[\beta s, s]_{\mathbb{T}}} w(\xi)\right)\right] \Delta s \\
& +\int_{\alpha\left(t_{0}\right)}^{\alpha(t)}\left[a(\gamma) h(w(\gamma))+b(\gamma) h\left(\max _{\xi \in[\beta \gamma, \gamma]_{\mathbb{T}}} w(\xi)\right)\right] \\
& \circ \alpha^{-1}(s) \widetilde{\Delta} s, \quad t \in \mathbb{T}_{0}, \\
w(t) \leq & \frac{\phi(t)}{k\left(t_{0}\right)}, \quad t \in\left[\beta \tau, t_{0}\right]_{\mathbb{T}} .
\end{aligned}
$$


Using Theorem 3.1 for (3.18) and (3.19), we get

$$
w(t) \leq H^{-1}\left(H(N)+\int_{t_{0}}^{t}\left[p(s)+q(s)+a(s) \alpha^{\Delta}(s)+b(s) \alpha^{\Delta}(s)\right] \Delta s\right), \quad t \in \mathbb{T}_{0},
$$

which results in (3.13). This completes the proof.

In the case when the function $k(t)$ involved in the right part of inequality (3.11) is not a monotonic function, we obtain the following result.

Theorem 3.3 Let the following conditions be satisfied:

(i) The conditions (i)-(ii) of Theorem 3.1 are satisfied.

(ii) The function $\phi \in C_{\mathrm{rd}}\left([\beta \tau, \infty)_{\mathbb{T}}, \mathbb{R}_{+}\right)$with $\max _{s \in\left[\beta \tau, t_{0}\right]_{\mathbb{T}}} \phi(s)>0$, where $0<\beta<1$ and $\tau=\min \left\{t_{0}, \alpha\left(t_{0}\right)\right\}$.

(iii) The function $h \in C\left(\mathbb{R}_{+}, \mathbb{R}_{+}\right)$and $h \in \Omega$.

(iv) The function $u \in C_{\mathrm{rd}}\left([\beta \tau, \infty)_{\mathbb{T}}, \mathbb{R}_{+}\right)$and satisfies the inequalities

$$
\begin{aligned}
u(t) \leq & \phi(t)+\int_{t_{0}}^{t}\left[p(s) h(u(s))+q(s) h\left(\max _{\xi \in[\beta s, s]_{\mathbb{T}}} u(\xi)\right)\right] \Delta s \\
& +\int_{\alpha\left(t_{0}\right)}^{\alpha(t)}\left[a(\gamma) h(u(\gamma))+b(\gamma) h\left(\max _{\xi \in[\beta \gamma, \gamma]_{\mathbb{T}}} u(\xi)\right)\right] \\
& \circ \alpha^{-1}(s) \widetilde{\Delta} s, \quad t \in \mathbb{T}_{0}, \\
u(t) \leq & \phi(t), \quad t \in\left[\beta \tau, t_{0}\right]_{\mathbb{T}} .
\end{aligned}
$$

Then, for all $t \in \mathbb{T}_{0}$ satisfying

$$
H(1)+\int_{t_{0}}^{t}\left[p(s)+q(s)+a(s) \alpha^{\Delta}(s)+b(s) \alpha^{\Delta}(s)\right] \Delta s \in \operatorname{Dom}\left(H^{-1}\right),
$$

we have

$$
u(t) \leq \phi(t)+f(t) H^{-1}\left(H(1)+\int_{t_{0}}^{t}\left[p(s)+q(s)+a(s) \alpha^{\Delta}(s)+b(s) \alpha^{\Delta}(s)\right] \Delta s\right),
$$

where $H(x)$ is defined by (3.5) and

$$
\begin{aligned}
f(t)= & \max _{s \in\left[\beta \tau, t_{0}\right]_{\mathbb{T}}} \phi(s)+\int_{t_{0}}^{t}\left[p(s) h(\phi(s))+q(s) h\left(\max _{\xi \in[\beta s, s]_{\mathbb{T}}} \phi(\xi)\right)\right] \Delta s \\
& +\int_{\alpha\left(t_{0}\right)}^{\alpha(t)}\left[a(\gamma) h(\phi(\gamma))+b(\gamma) h\left(\max _{\xi \in[\beta \gamma, \gamma]_{\mathbb{T}}} \phi(\xi)\right)\right] \circ \alpha^{-1}(s) \widetilde{\Delta} s, \quad t \in \mathbb{T}_{0} .
\end{aligned}
$$

Proof Let us define a function $z:[\beta \tau, \infty)_{\mathbb{T}} \rightarrow \mathbb{R}_{+}$by

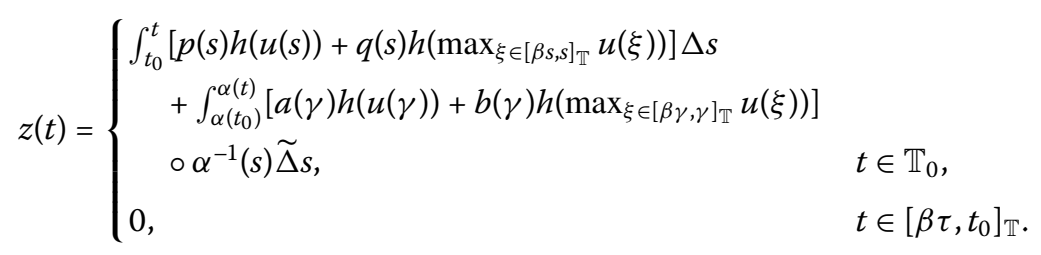


Therefore,

$$
u(t) \leq \phi(t)+z(t), \quad t \in[\beta \tau, \infty)_{\mathbb{T}} .
$$

From the definition of the function $z(t)$, it follows that

$$
\begin{aligned}
z(t) \leq & \int_{t_{0}}^{t}\left\{p(s) h(\phi(s)+z(s))+q(s) h\left(\max _{\xi \in[\beta s, s]_{\mathbb{T}}} \phi(\xi)+\max _{\xi \in[\beta s, s]_{\mathbb{T}}} z(\xi)\right)\right\} \Delta s \\
& +\int_{\alpha\left(t_{0}\right)}^{\alpha(t)}\{a(\gamma) h(\phi(\gamma)+z(\gamma)) \\
& \left.+b(\gamma) h\left(\max _{\xi \in[\beta \gamma, \gamma]_{\mathbb{T}}} \phi(\xi)+\max _{\xi \in[\beta \gamma, \gamma]_{\mathbb{T}}} z(\xi)\right)\right\} \circ \alpha^{-1}(s) \widetilde{\Delta} s \\
\leq & f(t)+\int_{t_{0}}^{t}\left\{p(s) h(z(s))+q(s) h\left(\max _{\xi \in[\beta s, s]_{\mathbb{T}}} z(\xi)\right)\right\} \Delta s \\
& +\int_{\alpha\left(t_{0}\right)}^{\alpha(t)}\left\{a(\gamma) h(z(\gamma))+b(\gamma) h\left(\max _{\xi \in[\beta \gamma, \gamma]_{\mathbb{T}}} z(\xi)\right)\right\} \circ \alpha^{-1}(s) \widetilde{\Delta} s, \quad t \in \mathbb{T}_{0}, \\
z(t) \leq & \phi(t), \quad t \in\left[\beta \tau, t_{0}\right]_{\mathbb{T}},
\end{aligned}
$$

where the function $f(t)$ is defined in (3.23).

Since the function $f(t): \mathbb{T}_{0} \rightarrow(0, \infty)$ is nondecreasing and $f\left(t_{0}\right)=\max _{s \in\left[\beta \tau, t_{0}\right]_{\mathbb{T}}} \phi(s)$, by using Theorem 3.2 for (3.26) and (3.27), we get

$$
z(t) \leq f(t) H^{-1}\left(H(1)+\int_{t_{0}}^{t}\left[p(s)+q(s)+a(s) \alpha^{\Delta}(s)+b(s) \alpha^{\Delta}(s)\right] \Delta s\right), \quad t \in \mathbb{T}_{0},
$$

which results in (3.22). This completes the proof.

Now we will consider an inequality in which the unknown function into the left part is presented in a power.

Theorem 3.4 Let the following conditions be fulfilled:

(i) The conditions (i)-(iii) of Theorem 3.1 and (iii) of Theorem 3.3 are satisfied.

(ii) The function $k \in C_{\mathrm{rd}}\left(\mathbb{T}_{0},(0, \infty)\right)$ is nondecreasing and the following inequality

$$
L:=\max _{s \in\left[\beta \tau, t_{0}\right]_{\mathbb{T}}} \phi(s) \leq \sqrt[n]{k\left(t_{0}\right)}, \quad n>1
$$

holds.

(iii) The function $u \in C_{\mathrm{rd}}\left([\beta \tau, \infty)_{\mathbb{T}}, \mathbb{R}_{+}\right)$and satisfies the inequalities

$$
\begin{aligned}
u^{n}(t) \leq & k(t)+\int_{t_{0}}^{t}\left[p(s) h(u(s))+q(s) h\left(\max _{\xi \in[\beta s,]_{\mathbb{T}}} u(\xi)\right)\right] \Delta s \\
& +\int_{\alpha\left(t_{0}\right)}^{\alpha(t)}\left[a(\gamma) h(u(\gamma))+b(\gamma) h\left(\max _{\xi \in[\beta \gamma, \gamma]_{\mathbb{T}}} u(\xi)\right)\right] \\
& \circ \alpha^{-1}(s) \widetilde{\Delta} s, \quad t \in \mathbb{T}_{0}, \\
u(t) \leq & \phi(t), \quad t \in\left[\beta \tau, t_{0}\right]_{\mathbb{T}} .
\end{aligned}
$$


Then, for all $t \in \mathbb{T}_{0}$ satisfying

$$
H(1)+\int_{t_{0}}^{t}\left[p(s)+q(s)+a(s) \alpha^{\Delta}(s)+b(s) \alpha^{\Delta}(s)\right] \Delta s \in \operatorname{Dom}\left(H^{-1}\right),
$$

we have

$$
\begin{aligned}
u(t) \leq & \frac{1}{n} c^{\frac{1-n}{n}} k(t)+\frac{n-1}{n} c^{\frac{1}{n}}+(L+g(t)) H^{-1}(H(1) \\
& \left.+\int_{t_{0}}^{t}\left[p(s)+q(s)+a(s) \alpha^{\Delta}(s)+b(s) \alpha^{\triangle}(s)\right] \Delta s\right),
\end{aligned}
$$

where

$$
\begin{aligned}
g(t)= & \int_{t_{0}}^{t}\left[p(s) h(w(s))+q(s) h\left(\max _{\xi \in[\beta s, s]_{\mathbb{T}}} w(\xi)\right)\right] \Delta s \\
& +\int_{\alpha\left(t_{0}\right)}^{\alpha(t)}\left[a(\gamma) h(w(\gamma))+b(\gamma) h\left(\max _{\xi \in[\beta \gamma, \gamma]_{\mathbb{T}}} w(\xi)\right)\right] \circ \alpha^{-1}(s) \widetilde{\Delta} s,
\end{aligned}
$$

with

$$
w(t)= \begin{cases}\frac{1}{n} c^{\frac{1-n}{n}} k(t)+\frac{n-1}{n} c^{\frac{1}{n}}, & t \in \mathbb{T}_{0}, \\ \frac{1}{n} c^{\frac{1-n}{n}} k\left(t_{0}\right)+\frac{n-1}{n} c^{\frac{1}{n}}, & t \in\left[\beta \tau, t_{0}\right]_{\mathbb{T}}\end{cases}
$$

for any constant $c \geq 1$.

Proof Define a function $z:[\beta \tau, \infty)_{\mathbb{T}} \rightarrow \mathbb{R}_{+}$by

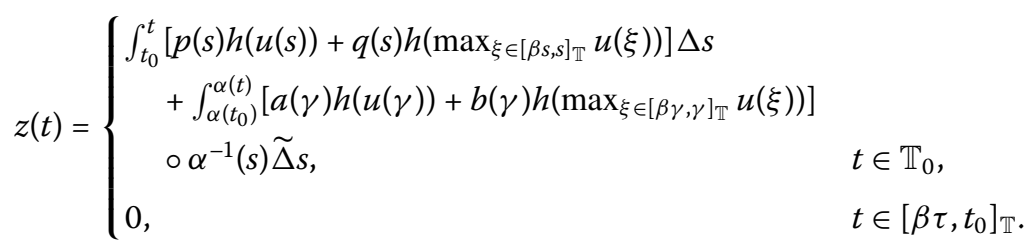

It follows from inequality (3.29) for $t \in \mathbb{T}_{0}$ that

$$
u(t) \leq[k(t)+z(t)]^{\frac{1}{n}} .
$$

Using Lemma 2.2, for any $c \geq 1$, we obtain

$$
\begin{aligned}
u(t) & \leq \frac{1}{n} c^{\frac{1-n}{n}}[k(t)+z(t)]+\frac{n-1}{n} c^{\frac{1}{n}} \\
& =\frac{1}{n} c^{\frac{1-n}{n}} k(t)+\frac{n-1}{n} c^{\frac{1}{n}}+\frac{1}{n} c^{\frac{1-n}{n}} z(t) \\
& =w(t)+\frac{1}{n} c^{\frac{1-n}{n}} z(t), \quad t \in \mathbb{T}_{0} .
\end{aligned}
$$

From inequality (3.28) and applying Lemma 2.2, for any $c \geq 1$, we have

$$
\sqrt[n]{k\left(t_{0}\right)} \leq \frac{1}{n} c^{\frac{1-n}{n}} k\left(t_{0}\right)+\frac{n-1}{n} c^{\frac{1}{n}}
$$


Indeed, by using inequality (3.36), we have for $t \in\left[\beta \tau, t_{0}\right]_{\mathbb{T}}$

$$
u(t) \leq \phi(t) \leq \phi(t)+\frac{1}{n} c^{\frac{1-n}{n}} z(t) \leq w(t)+\frac{1}{n} c^{\frac{1-n}{n}} z(t)
$$

where $w(t)$ is defined by (3.33).

Now we define a nondecreasing function $v: \mathbb{T}_{0} \rightarrow(0, \infty)$ by $v(t)=L+g(t)$, where $L$ and $g(t)$ are defined by (3.28) and (3.32), respectively.

From the definition of the function $z(t)$, it follows that

$$
\begin{aligned}
z(t) \leq & \int_{t_{0}}^{t}\left\{p(s) h\left(w(s)+\frac{1}{n} c^{\frac{1-n}{n}} z(s)\right)\right. \\
& \left.+q(s) h\left(\max _{\xi \in[\beta s, s]_{\mathbb{T}}} w(\xi)+\frac{1}{n} c^{\frac{1-n}{n}} \max _{\xi \in[\beta s, s] \mathbb{T}} z(\xi)\right)\right\} \Delta s \\
& +\int_{\alpha\left(t_{0}\right)}^{\alpha(t)}\left\{a(\gamma) h\left(w(\gamma)+\frac{1}{n} c^{\frac{1-n}{n}} z(\gamma)\right)\right. \\
& \left.+b(\gamma) h\left(\max _{\xi \in[\beta \gamma, \gamma]_{\mathbb{T}}} w(\xi)+\frac{1}{n} c^{\frac{1-n}{n}} \max _{\xi \in[\beta \gamma, \gamma]_{\mathbb{T}}} z(\xi)\right)\right\} \circ \alpha^{-1}(s) \widetilde{\Delta} s \\
\leq & v(t)+\int_{t_{0}}^{t}\left[p(s) h\left(\frac{1}{n} c^{\frac{1-n}{n}} z(s)\right)+q(s) h\left(\max _{\xi \in[\beta s, s]_{\mathbb{T}}} \frac{1}{n} c^{\frac{1-n}{n}} z(\xi)\right)\right] \Delta s \\
& +\int_{\alpha\left(t_{0}\right)}^{\alpha(t)}\left[a(\gamma) h\left(\frac{1}{n} c^{\frac{1-n}{n}} z(\gamma)\right)+b(\gamma) h\left(\max _{\xi \in[\beta \gamma, \gamma]_{\mathbb{T}}} \frac{1}{n} c^{\frac{1-n}{n}} z(\xi)\right)\right] \\
& \circ \alpha^{-1}(s) \widetilde{\Delta} s, \quad t \in \mathbb{T}_{0}, \\
z(t) \leq & \phi(t), \quad t \in\left[\beta \tau, t_{0}\right]_{\mathbb{T}} .
\end{aligned}
$$

From inequalities (3.38) and (3.39), we get for $c \geq 1, n>1$

$$
\begin{aligned}
\frac{1}{n} c^{\frac{1-n}{n}} z(t) \leq & v(t)+\int_{t_{0}}^{t}\left[p(s) h\left(\frac{1}{n} c^{\frac{1-n}{n}} z(s)\right)+q(s) h\left(\max _{\xi \in[\beta s, s]_{\mathbb{T}}} \frac{1}{n} c^{\frac{1-n}{n}} z(\xi)\right)\right] \Delta s \\
& +\int_{\alpha\left(t_{0}\right)}^{\alpha(t)}\left[a(\gamma) h\left(\frac{1}{n} c^{\frac{1-n}{n}} z(\gamma)\right)+b(\gamma) h\left(\max _{\xi \in[\beta \gamma, \gamma]_{\mathbb{T}}} \frac{1}{n} c^{\frac{1-n}{n}} z(\xi)\right)\right] \\
& \circ \alpha^{-1}(s) \widetilde{\Delta} s, \quad t \in \mathbb{T}_{0}, \\
\frac{1}{n} c^{\frac{1-n}{n}} z(t) \leq & \phi(t), \quad t \in\left[\beta \tau, t_{0}\right]_{\mathbb{T}} .
\end{aligned}
$$

Applying Theorem 3.2 for (3.40) and (3.41), we obtain

$$
\frac{1}{n} c^{\frac{1-n}{n}} z(t) \leq v(t) H^{-1}\left(H(1)+\int_{t_{0}}^{t}\left[p(s)+q(s)+a(s) \alpha^{\Delta}(s)+b(s) \alpha^{\Delta}(s)\right] \Delta s\right), \quad t \in \mathbb{T}_{0},
$$

which results in (3.31). This completes the proof.

Next we will consider an inequality which has powers on both sizes.

Theorem 3.5 Let the following conditions be fulfilled:

(i) The conditions (i)-(iii) of Theorem 3.1 and (iii) if Theorem 3.3 are satisfied. 
(ii) The function $k \in C_{\mathrm{rd}}\left(\mathbb{T}_{0},(0, \infty)\right)$ is nondecreasing and the following inequality

$$
K:=\max _{s \in\left[\beta \tau, t_{0}\right]_{\mathbb{T}}}\left\{\phi^{\varepsilon}(s), \phi^{l}(s)\right\} \leq \frac{m}{n} c^{\frac{m-n}{n}} k\left(t_{0}\right)+\frac{n-1}{n} c^{\frac{m}{n}}
$$

holds for any constant $c \geq 1$ and $n \geq m \geq l \geq \delta \geq \varepsilon>1$.

(iii) The function $u \in C_{\mathrm{rd}}\left([\beta \tau, \infty)_{\mathbb{T}}, \mathbb{R}_{+}\right)$and satisfies the inequalities

$$
\begin{aligned}
u^{n}(t) \leq & k(t)+\int_{t_{0}}^{t}\left[p(s) h\left(u^{m}(s)\right)+q(s) h\left(\max _{\xi \in[\beta s, s]]_{\mathbb{T}}} u^{l}(\xi)\right)\right] \Delta s \\
& +\int_{\alpha\left(t_{0}\right)}^{\alpha(t)}\left[a(\gamma) h\left(u^{\delta}(\gamma)\right)+b(\gamma) h\left(\max _{\xi \in[\beta \gamma, \gamma]_{\mathbb{T}}} u^{\varepsilon}(\xi)\right)\right] \\
& \circ \alpha^{-1}(s) \widetilde{\Delta} s, \quad t \in \mathbb{T}_{0}, \\
u(t) \leq & \phi(t), \quad t \in\left[\beta \tau, t_{0}\right]_{\mathbb{T}} .
\end{aligned}
$$

Then, for all $t \in \mathbb{T}_{0}$ satisfying

$$
H(1)+\int_{t_{0}}^{t}\left[p(s)+q(s)+a(s) \alpha^{\triangle}(s)+b(s) \alpha^{\triangle}(s)\right] \Delta s \in \operatorname{Dom}\left(H^{-1}\right),
$$

we have

$$
\begin{aligned}
u(t) \leq & \frac{1}{n} c^{\frac{1-n}{n}} k(t)+\frac{n-1}{n} c^{\frac{1}{n}}+\frac{1}{m} c^{\frac{1-m}{n}}(K+\lambda(t)) H^{-1}(H(1) \\
& \left.+\int_{t_{0}}^{t}\left[p(s)+q(s)+a(s) \alpha^{\triangle}(s)+b(s) \alpha^{\triangle}(s)\right] \Delta s\right)
\end{aligned}
$$

where

$$
\begin{aligned}
\lambda(t)= & \int_{t_{0}}^{t}\left[p(s) h(\bar{w}(s))+q(s) h\left(\max _{\xi \in[\beta s, s]_{\mathbb{T}}} \bar{w}(\xi)\right)\right] \Delta s \\
& +\int_{\alpha\left(t_{0}\right)}^{\alpha(t)}\left[a(\gamma) h(\bar{w}(\gamma))+b(\gamma) h\left(\max _{\xi \in[\beta \gamma, \gamma]_{\mathbb{T}}} \bar{w}(\xi)\right)\right] \circ \alpha^{-1}(s) \widetilde{\Delta} s,
\end{aligned}
$$

with

$$
\bar{w}(t)= \begin{cases}\frac{m}{n} c^{\frac{m-n}{n}} k(t)+\frac{n-1}{n} c^{\frac{m}{n}}, & t \in \mathbb{T}_{0}, \\ \frac{m}{n} c^{\frac{m-n}{n}} k\left(t_{0}\right)+\frac{n-1}{n} c^{\frac{m}{n}}, & t \in\left[\beta \tau, t_{0}\right]_{\mathbb{T}}\end{cases}
$$

Proof We define a function $z:[\beta \tau, \infty)_{\mathbb{T}} \rightarrow \mathbb{R}_{+}$by

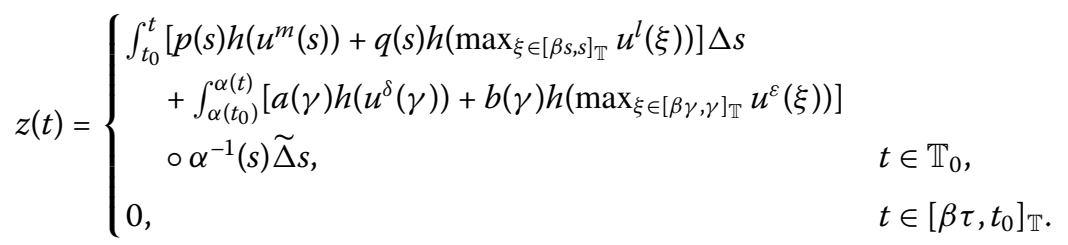


From inequality (3.43) we have for $t \in \mathbb{T}_{0}$

$$
\begin{aligned}
& u(t) \leq[k(t)+z(t)]^{\frac{1}{n}}, \\
& u^{l}(t) \leq[k(t)+z(t)]^{\frac{l}{n}}, \\
& u^{m}(t) \leq[k(t)+z(t)]^{\frac{m}{n}}, \\
& u^{\delta}(t) \leq[k(t)+z(t)]^{\frac{\delta}{n}}, \\
& u^{\varepsilon}(t) \leq[k(t)+z(t)]^{\frac{\varepsilon}{n}} .
\end{aligned}
$$

By using Lemma 2.2, for any $c \geq 1$, we obtain

$$
\begin{aligned}
u(t) & \leq \frac{1}{n} c^{\frac{1-n}{n}} k(t)+\frac{n-1}{n} c^{\frac{1}{n}}+\frac{1}{n} c^{\frac{1-n}{n}} z(t), \quad t \in \mathbb{T}_{0}, \\
u^{\varepsilon}(t) & \leq \frac{\varepsilon}{n} c^{\frac{\varepsilon-n}{n}} k(t)+\frac{n-\varepsilon}{n} c^{\frac{\varepsilon}{n}}+\frac{\varepsilon}{n} c^{\frac{\varepsilon-n}{n}} z(t) \\
& \leq \bar{w}(t)+\frac{m}{n} c^{\frac{m-n}{n}} z(t), \quad t \in \mathbb{T}_{0}, \\
u^{\delta}(t) & \leq \frac{\delta}{n} c^{\frac{\delta-n}{n}} k(t)+\frac{n-\delta}{n} c^{\frac{\delta}{n}}+\frac{\delta}{n} c^{\frac{\delta-n}{n}} z(t) \\
& \leq \bar{w}(t)+\frac{m}{n} c^{\frac{m-n}{n}} z(t), \quad t \in \mathbb{T}_{0}, \\
u^{l}(t) & \leq \frac{l}{n} c^{\frac{l-n}{n}} k(t)+\frac{n-l}{n} c^{\frac{l}{n}}+\frac{l}{n} c^{\frac{l-n}{n}} z(t) \\
& \leq \bar{w}(t)+\frac{m}{n} c^{\frac{m-n}{n}} z(t), \quad t \in \mathbb{T}_{0}, \\
u^{m}(t) & \leq \frac{m}{n} c^{\frac{m-n}{n}} k(t)+\frac{n-m}{n} c^{\frac{m}{n}}+\frac{m}{n} c^{\frac{m-n}{n}} z(t) \\
& \leq \bar{w}(t)+\frac{m}{n} c^{\frac{m-n}{n}} z(t), \quad t \in \mathbb{T}_{0} .
\end{aligned}
$$

Moreover, we have

$$
\begin{aligned}
u^{\varepsilon}(t) & \leq \phi^{\varepsilon}(t) \leq \phi^{\varepsilon}(t)+\frac{m}{n} c^{\frac{m-n}{n}} z(t) \\
& \leq \bar{w}(t)+\frac{m}{n} c^{\frac{m-n}{n}} z(t), \quad t \in\left[\beta \tau, t_{0}\right]_{\mathbb{T}}
\end{aligned}
$$

and

$$
\begin{aligned}
u^{l}(t) & \leq \phi^{l}(t) \leq \phi^{l}(t)+\frac{m}{n} c^{\frac{m-n}{n}} z(t) \\
& \leq \bar{w}(t)+\frac{m}{n} c^{\frac{m-n}{n}} z(t), \quad t \in\left[\beta \tau, t_{0}\right]_{\mathbb{T}},
\end{aligned}
$$

where $\bar{w}(t)$ is defined by (3.47). From the definition of the function $z(t)$, it follows that

$$
\begin{aligned}
z(t) \leq & \int_{t_{0}}^{t}\left\{p(s) h\left(\bar{w}(s)+\frac{m}{n} c^{\frac{m-n}{n}} z(s)\right)\right. \\
& \left.+q(s) h\left(\max _{\xi \in[\beta s, s]_{\mathbb{T}}} \bar{w}(\xi)+\max _{\xi \in[\beta s, s]_{\mathbb{T}}} \frac{m}{n} c^{\frac{m-n}{n}} z(\xi)\right)\right\} \Delta s
\end{aligned}
$$




$$
\begin{aligned}
+ & \int_{\alpha\left(t_{0}\right)}^{\alpha(t)}\left\{a(\gamma) h\left(\bar{w}(\gamma)+\frac{m}{n} c^{\frac{m-n}{n}} z(\gamma)\right)\right. \\
& \left.+b(\gamma) h\left(\max _{\xi \in[\beta \gamma, \gamma]_{\mathbb{T}}} \bar{w}(\xi)+\max _{\xi \in[\beta \gamma, \gamma]_{\mathbb{T}}} \frac{m}{n} c^{\frac{m-n}{n}} z(\xi)\right)\right\} \circ \alpha^{-1}(s) \widetilde{\Delta} s \\
\leq & \rho(t)+\int_{t_{0}}^{t}\left[p(s) h\left(\frac{m}{n} c^{\frac{m-n}{n}} z(s)\right)+q(s) h\left(\max _{\xi \in[\beta s, s]_{\mathbb{T}}} \frac{m}{n} c^{\frac{m-n}{n}} z(\xi)\right)\right] \Delta s \\
& +\int_{\alpha\left(t_{0}\right)}^{\alpha(t)}\left[a(\gamma) h\left(\frac{m}{n} c^{\frac{m-n}{n}} z(\gamma)\right)+b(\gamma) h\left(\max _{\xi \in[\beta \gamma, \gamma]_{\mathbb{T}}} \frac{m}{n} c^{\frac{m-n}{n}} z(\xi)\right)\right] \\
& \circ \alpha^{-1}(s) \widetilde{\Delta} s, \quad t \in \mathbb{T}_{0}, \\
z(t) \leq & \phi(t), \quad t \in\left[\beta \tau, t_{0}\right]_{\mathbb{T}} .
\end{aligned}
$$

From inequalities (3.56) and (3.57), we have

$$
\begin{aligned}
\frac{m}{n} c^{\frac{m-n}{n}} z(t) \leq & \rho(t)+\int_{t_{0}}^{t}\left[p(s) h\left(\frac{m}{n} c^{\frac{m-n}{n}} z(s)\right)+q(s) h\left(\max _{\xi \in[\beta s, s]_{\mathbb{T}}} \frac{m}{n} c^{\frac{m-n}{n}} z(\xi)\right)\right] \Delta s \\
& +\int_{\alpha\left(t_{0}\right)}^{\alpha(t)}\left[a(\gamma) h\left(\frac{m}{n} c^{\frac{m-n}{n}} z(\gamma)\right)+b(\gamma) h\left(\max _{\xi \in[\beta \gamma, \gamma]_{\mathbb{T}}} \frac{m}{n} c^{\frac{m-n}{n}} z(\xi)\right)\right] \\
& \circ \alpha^{-1}(s) \widetilde{\Delta} s, \quad t \in \mathbb{T}_{0}, \\
\frac{m}{n} c^{\frac{m-n}{n}} z(t) \leq & \phi(t), \quad t \in\left[\beta \tau, t_{0}\right]_{\mathbb{T}},
\end{aligned}
$$

where a nondecreasing function $\rho(t): \mathbb{T}_{0} \rightarrow(0, \infty)$ is defined by $\rho(t):=K+\lambda(t)$, where $K$ and $\lambda(t)$ are defined in (3.42) and (3.46), respectively.

Applying Theorem 3.2 for (3.58) and (3.59), we obtain

$$
\frac{m}{n} c^{\frac{m-n}{n}} z(t) \leq \rho(t) H^{-1}\left(H(1)+\int_{t_{0}}^{t}\left[p(s)+q(s)+a(s) \alpha^{\triangle}(s)+b(s) \alpha^{\triangle}(s)\right] \Delta s\right), \quad t \in \mathbb{T}_{0}
$$

which results in (3.45). This completes the proof.

In the case when the unknown function is involved nonlinearly in the left part of the inequality, we obtain the following result.

Theorem 3.6 Let the following conditions be fulfilled:

(i) The conditions (i)-(iv) of Theorem 3.1 are satisfied.

(ii) The function $\Psi \in C\left(\mathbb{R}_{+}, \mathbb{R}_{+}\right)$is strictly increasing, $\lim _{t \rightarrow \infty} \Psi(t)=\infty$.

(iii) The function $u \in C_{\mathrm{rd}}\left([\beta \tau, \infty)_{\mathbb{T}}, \mathbb{R}_{+}\right)$and satisfies the inequalities

$$
\begin{aligned}
& \Psi(u(t)) \leq k+\int_{t_{0}}^{t}\left[p(s) h(u(s))+q(s) h\left(\max _{\xi \in[\beta s, s]_{\mathbb{T}}} u(\xi)\right)\right] \Delta s \\
& +\int_{\alpha\left(t_{0}\right)}^{\alpha(t)}\left[a(\gamma) h(u(\gamma))+b(\gamma) h\left(\max _{\xi \in[\beta \gamma, \gamma]_{\mathbb{T}}} u(\xi)\right)\right] \\
& \circ \alpha^{-1}(s) \widetilde{\Delta} s, \quad t \in \mathbb{T}_{0}, \\
& u(t) \leq \phi(t), \quad t \in\left[\beta \tau, t_{0}\right]_{\mathbb{T}},
\end{aligned}
$$

where $k \geq 0$. 
Then, for all $t \in \mathbb{T}_{0}$ satisfying

$$
\widetilde{H}(\Psi(P))+\int_{t_{0}}^{t}\left[p(s)+q(s)+a(s) \alpha^{\Delta}(s)+b(s) \alpha^{\Delta}(s)\right] \Delta s \in \operatorname{Dom}\left(\widetilde{H}^{-1}\right)
$$

and

$$
\widetilde{H}^{-1}\left(\widetilde{H}(\Psi(P))+\int_{t_{0}}^{t}\left[p(s)+q(s)+a(s) \alpha^{\Delta}(s)+b(s) \alpha^{\Delta}(s)\right] \Delta s\right) \in \operatorname{Dom}\left(\Psi^{-1}\right),
$$

we have

$$
u(t) \leq \Psi^{-1}\left\{\widetilde{H}^{-1}\left(\widetilde{H}(\Psi(P))+\int_{t_{0}}^{t}\left[p(s)+q(s)+a(s) \alpha^{\Delta}(s)+b(s) \alpha^{\Delta}(s)\right] \Delta s\right)\right\},
$$

where

$$
P=\max \left\{\Psi^{-1}(k), \max _{s \in\left[\beta \tau, t_{0}\right]_{\mathbb{T}}} \phi(s)\right\}
$$

and

$$
\widetilde{H}(x)=\int_{x_{0}}^{x} \frac{1}{h\left(\Psi^{-1}(r)\right)} d r, \quad x_{0}>0,
$$

where $\widetilde{H}(\infty)=\infty$, and $\widetilde{H}^{-1}$ is the inverse of $\widetilde{H}$.

Proof Define a function $z:[\beta \tau, \infty)_{\mathbb{T}} \rightarrow \mathbb{R}_{+}$by

$$
z(t)= \begin{cases}\Psi(P)+\int_{t_{0}}^{t}\left[p(s) h(u(s))+q(s) h\left(\max _{\xi \in[\beta s, s]_{\mathbb{T}}} u(\xi)\right)\right] \Delta s & \\ \quad+\int_{\alpha\left(t_{0}\right)}^{\alpha(t)}\left[a(\gamma) h(u(\gamma))+b(\gamma) h\left(\max _{\xi \in[\beta \gamma, \gamma]_{\mathbb{T}}} u(\xi)\right)\right] \circ \alpha^{-1}(s) \widetilde{\Delta} s, & t \in \mathbb{T}_{0}, \\ \Psi(P), & t \in\left[\beta \tau, t_{0}\right]_{\mathbb{T}},\end{cases}
$$

where $P$ is defined by (3.63). Note that the function $z(t)$ is nondecreasing.

It follows that the inequality

$$
u(t) \leq \Psi^{-1}(z(t)), \quad t \in[\beta \tau, \infty)_{\mathbb{T}}
$$

holds. Therefore, for $t \in \mathbb{T}_{0}$ and $s \in\left[t_{0}, t\right]_{\mathbb{T}}$, we have

$$
\max _{\xi \in[\beta s, s]_{\mathbb{T}}} u(\xi) \leq \max _{\xi \in[\beta s, s]_{\mathbb{T}}} \Psi^{-1}(z(\xi))=\Psi^{-1}(z(s))
$$

For $t \in \mathbb{T}_{0}$ and $s \in\left[\alpha\left(t_{0}\right), \alpha(t)\right]_{\widetilde{T}}$, we have

$$
\begin{aligned}
h\left(\max _{\xi \in[\beta \gamma, \gamma]]_{\mathbb{T}}} u(\xi)\right) \circ \alpha^{-1}(s) & \leq h\left(\max _{\xi \in[\beta \gamma, \gamma]]_{\mathbb{T}}} \Psi^{-1}(z(\xi))\right) \circ \alpha^{-1}(s) \\
& =h\left(\max _{\xi \in\left[\beta \alpha^{-1}(s), \alpha^{-1}(s)\right]_{\mathbb{T}}} \Psi^{-1}(z(\xi))\right) \\
& =h\left(\Psi^{-1}\left(z\left(\alpha^{-1}(s)\right)\right)\right) \\
& =h\left(\Psi^{-1}(z(\gamma))\right) \circ \alpha^{-1}(s) .
\end{aligned}
$$


Then, from the definition of $z(t)$ and the above analysis, we get for $t \in \mathbb{T}_{0}$ that

$$
\begin{aligned}
z(t) \leq & \Psi(P)+\int_{t_{0}}^{t}\left[p(s) h\left(\Psi^{-1}(z(s))\right)+q(s) h\left(\max _{\xi \in[\beta s, s]_{\mathbb{T}}} \Psi^{-1}(z(\xi))\right)\right] \Delta s \\
& +\int_{\alpha\left(t_{0}\right)}^{\alpha(t)}\left[a(\gamma) h\left(\Psi^{-1}(z(\gamma))\right)+b(\gamma) h\left(\max _{\xi \in[\beta \gamma, \gamma]_{\mathbb{T}}} \Psi^{-1}(z(\xi))\right)\right] \\
& \circ \alpha^{-1}(s) \widetilde{\Delta} s, \quad t \in \mathbb{T}_{0}, \\
z(t) \leq & \Psi(P), \quad t \in\left[\beta \tau, t_{0}\right]_{\mathbb{T}} .
\end{aligned}
$$

According to Theorem 3.1, from inequalities (3.65) and (3.66), we have

$$
z(t) \leq \widetilde{H}^{-1}\left(\widetilde{H}(\Psi(P))+\int_{t_{0}}^{t}\left[p(s)+q(s)+a(s) \alpha^{\Delta}(s)+b(s) \alpha^{\Delta}(s)\right] \Delta s\right), \quad t \in \mathbb{T}_{0},
$$

which results in (3.62). This completes the proof.

In the case when in place of the constant $k$ involved in Theorem 3.6 we have a function $k(t)$, we obtain the following result.

Theorem 3.7 Let the following conditions be fulfilled:

(i) The conditions (i)-(iii) of Theorem 3.1, (ii) of Theorem 3.2 and (ii) of Theorem 3.6 are satisfied.

(ii) The function $k \in C_{\mathrm{rd}}\left(\mathbb{T}_{0},[1, \infty)\right)$ is nondecreasing and the inequality $Q=\max _{s \in\left[\beta \tau, t_{0}\right]_{\mathbb{T}}} \phi(s) \leq \Psi^{-1}\left(k\left(t_{0}\right)\right)$ holds.

(iii) The function $u \in C_{\mathrm{rd}}\left([\beta \tau, \infty)_{\mathbb{T}}, \mathbb{R}_{+}\right)$and satisfies the inequalities

$$
\begin{aligned}
& \Psi(u(t)) \leq k(t)+\int_{t_{0}}^{t}\left[p(s) h(u(s))+q(s) h\left(\max _{\xi \in[\beta s, s]_{\mathbb{T}}} u(\xi)\right)\right] \Delta s \\
&+\int_{\alpha\left(t_{0}\right)}^{\alpha(t)}\left[a(\gamma) h(u(\gamma))+b(\gamma) h\left(\max _{\xi \in[\beta \gamma, \gamma]_{\mathbb{T}}} u(\xi)\right)\right] \\
& \circ \alpha^{-1}(s) \widetilde{\Delta} s, \quad t \in \mathbb{T}_{0}, \\
& u(t) \leq \phi(t), \quad t \in\left[\beta \tau, t_{0}\right]_{\mathbb{T}} .
\end{aligned}
$$

Then, for all $t \in \mathbb{T}_{0}$ satisfying

$$
\widetilde{H}(1)+\int_{t_{0}}^{t}\left[p(s)+q(s)+a(s) \alpha^{\Delta}(s)+b(s) \alpha^{\Delta}(s)\right] \Delta s \in \operatorname{Dom}\left(\widetilde{H}^{-1}\right)
$$

and

$$
k(t) \widetilde{H}^{-1}\left(\widetilde{H}(1)+\int_{t_{0}}^{t}\left[p(s)+q(s)+a(s) \alpha^{\Delta}(s)+b(s) \alpha^{\Delta}(s)\right] \Delta s\right) \in \operatorname{Dom}\left(\Psi^{-1}\right),
$$

we have

$$
u(t) \leq \Psi^{-1}\left\{k(t) \tilde{H}^{-1}\left(\widetilde{H}(1)+\int_{t_{0}}^{t}\left[p(s)+q(s)+a(s) \alpha^{\Delta}(s)+b(s) \alpha^{\Delta}(s)\right] \Delta s\right)\right\},
$$

where $\widetilde{H}(x)$ is defined by (3.64). 
Proof Define a function $z:[\beta \tau, \infty)_{\mathbb{T}} \rightarrow \mathbb{R}_{+}$by

$$
z(t)= \begin{cases}k(t)+\int_{t_{0}}^{t}\left[p(s) h(u(s))+q(s) h\left(\max _{\xi \in[\beta s, s]_{\mathbb{T}}} u(\xi)\right)\right] \Delta s & \\ \quad+\int_{\alpha\left(t_{0}\right)}^{\alpha(t)}\left[a(\gamma) h(u(\gamma))+b(\gamma) h\left(\max _{\xi \in[\beta \gamma, \gamma]_{\mathbb{T}}} u(\xi)\right)\right] \circ \alpha^{-1}(s) \widetilde{\Delta} s, & t \in \mathbb{T}_{0}, \\ k\left(t_{0}\right), & t \in\left[\beta \tau, t_{0}\right]_{\mathbb{T}} .\end{cases}
$$

Note that the function $z(t)$ is nondecreasing. It follows that the inequality

$$
u(t) \leq \Psi^{-1}(z(t)), \quad t \in[\beta \tau, \infty)_{\mathbb{T}}
$$

holds. Therefore, for $t \in \mathbb{T}_{0}$ and $s \in\left[t_{0}, t\right]_{\mathbb{T}}$, we have

$$
\max _{\xi \in[\beta s, s]_{\mathbb{T}}} u(\xi) \leq \max _{\xi \in[\beta s, s]_{\mathbb{T}}} \Psi^{-1}(z(\xi))=\Psi^{-1}(z(s))
$$

For $t \in \mathbb{T}_{0}$ and $s \in\left[\alpha\left(t_{0}\right), \alpha(t)\right] \widetilde{\mathbb{T}}$, we have

$$
\begin{aligned}
h\left(\max _{\xi \in[\beta \gamma, \gamma]_{\mathbb{T}}} u(\xi)\right) \circ \alpha^{-1}(s) & \leq h\left(\max _{\xi \in[\beta \gamma, \gamma]_{\mathbb{T}}} \Psi^{-1}(z(\xi))\right) \circ \alpha^{-1}(s) \\
& =h\left(\max _{\xi \in\left[\beta \alpha^{-1}(s), \alpha^{-1}(s)\right]_{\mathbb{T}}} \Psi^{-1}(z(\xi))\right) \\
& =h\left(\Psi^{-1}\left(z\left(\alpha^{-1}(s)\right)\right)\right) \\
& =h\left(\Psi^{-1}(z(\gamma))\right) \circ \alpha^{-1}(s) .
\end{aligned}
$$

Then, from the definition of $z(t)$ and the above analysis, we get for $t \in \mathbb{T}_{0}$ that

$$
\begin{aligned}
z(t) \leq & k(t)+\int_{t_{0}}^{t}\left[p(s) h\left(\Psi^{-1}(z(s))\right)+q(s) h\left(\max _{\xi \in[\beta s, s]_{\mathbb{T}}} \Psi^{-1}(z(\xi))\right)\right] \Delta s \\
& +\int_{\alpha\left(t_{0}\right)}^{\alpha(t)}\left[a(\gamma) h\left(\Psi^{-1}(z(\gamma))\right)+b(\gamma) h\left(\max _{\xi \in[\beta \gamma, \gamma]_{\mathbb{T}}} \Psi^{-1}(z(\xi))\right)\right] \\
& \circ \alpha^{-1}(s) \widetilde{\Delta} s, \quad t \in \mathbb{T}_{0}, \\
z(t) \leq & k\left(t_{0}\right), \quad t \in\left[\beta \tau, t_{0}\right]_{\mathbb{T}} .
\end{aligned}
$$

According to Theorem 3.2, from inequalities (3.70) and (3.71), we have

$$
z(t) \leq k(t) \widetilde{H}^{-1}\left(\widetilde{H}(1)+\int_{t_{0}}^{t}\left[p(s)+q(s)+a(s) \alpha^{\Delta}(s)+b(s) \alpha^{\Delta}(s)\right] \Delta s\right), \quad t \in \mathbb{T}_{0}
$$

which results in (3.69). This completes the proof.

\section{An application}

In this section, in order to illustrate our results, we consider the following first-order dynamic equation with 'maxima':

$$
x^{\triangleright}(t)=F\left(t, x(t), \max _{s \in[\beta t, t]_{\mathbb{T}}} x(s)\right), \quad t \in \mathbb{T}_{0},
$$


and initial condition

$$
x(t)=\phi(t), \quad t \in\left[\beta \tau, t_{0}\right]_{\mathbb{T}},
$$

where $F \in C_{\mathrm{rd}}\left(\mathbb{T}_{0} \times \mathbb{R} \times \mathbb{R}, \mathbb{R}\right), \phi \in C_{\mathrm{rd}}\left(\left[\beta t_{0}, t_{0}\right]_{\mathbb{T}}, \mathbb{R}\right), 0<\beta<1, \tau$ is a constant such that $\beta \tau \leq t_{0}$.

Corollary 4.1 Assume that:

$\left(\mathrm{H}_{1}\right)$ There exists a strictly increasing function $\alpha \in C_{\mathrm{rd}}\left(\mathbb{T}_{0}, \mathbb{R}_{+}\right)$such that $\alpha(\mathbb{T})=\widetilde{\mathbb{T}}$ is a time scale and $\min \left\{t_{0}, \alpha\left(t_{0}\right)\right\}=\tau$.

$\left(\mathrm{H}_{2}\right)$ There exist functions $A, B, C, D, \alpha^{\Delta} \in C_{\mathrm{rd}}\left(\mathbb{T}_{0}, \mathbb{R}_{+}\right)$and an integer $p>1$ such that for $t \in \mathbb{T}_{0}, u, v \in \mathbb{R}$,

$$
|F(t, u, v)| \leq\left(A(t)+B(t) \alpha^{\triangle}(t)\right) \sqrt[p]{|u|}+\left(C(t)+D(t) \alpha^{\triangle}(t)\right) \sqrt[p]{|v|} .
$$

Then the solution $x(t)$ of IVP (4.1)-(4.2) satisfies the following inequality:

$$
|x(t)| \leq\left\{M^{\frac{p-1}{p}}+\frac{p-1}{p} \int_{t_{0}}^{t}\left[A(s)+C(s)+B(s) \alpha^{\Delta}(s)+D(s) \alpha^{\Delta}(s)\right] \Delta s\right\}^{\frac{p}{p-1}}, \quad t \in \mathbb{T}_{0},
$$

where

$$
M=\max _{s \in\left[\beta \tau, t_{0}\right]_{\mathbb{T}}}|\phi(s)| .
$$

Proof It is easy to see that the solution $x(t)$ of IVP (4.1)-(4.2) satisfies the following equation:

$$
x(t)=\phi\left(t_{0}\right)+\int_{t_{0}}^{t} F\left(s, x(s), \max _{\xi \in[\beta s,]_{\mathbb{T}}} x(\xi)\right) \Delta s .
$$

Using the assumption $\left(\mathrm{H}_{2}\right)$, it follows from (4.5) that

$$
\begin{aligned}
|x(t)| \leq & \left|\phi\left(t_{0}\right)\right|+\int_{t_{0}}^{t}\left|F\left(s, x(s), \max _{\xi \in[\beta s, s]_{\mathbb{T}}} x(\xi)\right)\right| \Delta s \\
\leq & \left|\phi\left(t_{0}\right)\right|+\int_{t_{0}}^{t}\left[\left(A(s)+B(s) \alpha^{\Delta}(s)\right) \sqrt[p]{|x(s)|}\right. \\
& \left.+\left(C(s)+D(s) \alpha^{\Delta}(s)\right) \sqrt[p]{\left|\max _{\xi \in[\beta s, s]_{\mathbb{T}}} x(\xi)\right|}\right] \Delta s \\
\leq & \left|\phi\left(t_{0}\right)\right|+\int_{t_{0}}^{t}\left[A(s) \sqrt[p]{|x(s)|}+C(s) \sqrt[p]{\max _{\xi \in[\beta s, s]_{\mathbb{T}}}|x(\xi)|}\right] \Delta s \\
& +\int_{t_{0}}^{t}\left[B(s) \sqrt[p]{|x(s)|}+D(s) \sqrt[p]{\max _{\xi \in[\beta s, s] \mathbb{T}}|x(\xi)|}\right] \alpha^{\Delta}(s) \Delta s \\
= & \left|\phi\left(t_{0}\right)\right|+\int_{t_{0}}^{t}\left[A(s) \sqrt[p]{|x(s)|}+C(s) \sqrt[p]{\max _{\xi \in[\beta s, s]_{\mathbb{T}}}|x(\xi)|}\right] \Delta s \\
& +\int_{\alpha\left(t_{0}\right)}^{\alpha(t)}\left[B(\gamma) \sqrt[p]{|x(\gamma)|}+D(\gamma) \sqrt[p]{\max _{\xi \in[\beta \gamma, \gamma]_{\mathbb{T}}}|x(\xi)|}\right] \circ \alpha^{-1}(s) \widetilde{\Delta} s .
\end{aligned}
$$


Hence Corollary 4.1 yields the estimate

$$
|x(t)| \leq\left\{M^{\frac{p-1}{p}}+\frac{p-1}{p} \int_{t_{0}}^{t}\left[A(s)+C(s)+B(s) \alpha^{\triangle}(s)+D(s) \alpha^{\triangle}(s)\right] \Delta s\right\}^{\frac{p}{p-1}}, \quad t \in \mathbb{T}_{0} .
$$

Inequality (4.7) gives the bound on the solution $x(t)$ of IVP (4.1)-(4.2).

Example 4.1 Consider the following first-order dynamic equation with 'maxima' on time scale $\mathbb{T}=\left\{2^{n}: n \in \mathbb{Z}\right\} \cup\{0\}$ ( $\mathbb{Z}$ stands for the integer set):

$$
\left\{\begin{aligned}
x^{\Delta}(t)= & \frac{1}{2} \tan ^{-1}\left(\left(2+8 t^{2}\right) \sqrt[3]{x(t)}\right) \\
& +2 \sin \left(\left(e^{2 t}+4 \cos ^{2}(\pi t)\right) \sqrt[3]{\max _{s \in\left[\frac{1}{16} t, t\right]_{\mathbb{T}}} x(s)}\right), \quad t \in \mathbb{T}_{0}
\end{aligned}\right.
$$

where $\mathbb{T}_{0}=[2, \infty) \cap \mathbb{T}$.

Here $\phi(t)=3, \beta=1 / 16, p=3, F\left(t, x(t), \max _{s \in[\beta t, t]} x(s)\right)=\left(\tan ^{-1}\left(\left(2+8 t^{2}\right) \times \sqrt[3]{x(t)}\right)\right) / 2+$ $2 \sin \left(\left(e^{2 t}+4 \cos ^{2}(\pi t)\right) \sqrt[3]{\max _{\left.s \in\left[\frac{1}{16} t, t\right]\right]_{\mathbb{T}}} x(s)}\right), t_{0}=2, \tau=2$.

By choosing $\alpha(t)=4 t$, we can show that $\alpha(\mathbb{T})=\widetilde{\mathbb{T}} \subseteq \mathbb{T}$ and $\min \left\{t_{0}, \alpha\left(t_{0}\right)\right\}=2$. Clearly,

$$
\begin{aligned}
\left|F\left(t, x(t), \max _{s \in[\beta t, t]_{\mathbb{T}}} x(s)\right)\right|= & \frac{1}{2} \tan ^{-1}\left(\left(2+8 t^{2}\right) \sqrt[3]{x(t)}\right) \\
& +2 \sin \left(\left(e^{2 t}+4 \cos ^{2}(\pi t)\right) \sqrt[3]{\max _{s \in\left[\frac{1}{16} t, t\right]_{\mathbb{T}}} x(s)}\right) \mid \\
\leq & \left(1+4 t^{2}\right) \sqrt[3]{|x(t)|}+\left(2 e^{2 t}+8 \cos ^{2}(\pi t)\right) \sqrt[3]{\left|\max _{s \in\left[\frac{1}{16} t, t\right]_{\mathbb{T}}} x(s)\right|}
\end{aligned}
$$

and

$$
\max _{s \in[(1 /(8)),(2)]_{\mathbb{T}}}|\phi(s)|=3
$$

On the other hand, we have $\alpha^{\triangle}(t)=4$. Set $A(t)=1, B(t)=t^{2}, C(t)=2 e^{2 t}$ and $D(t)=$ $2 \cos ^{2}(\pi t)$. Hence, Corollary 4.1 yields the estimate

$$
|x(t)| \leq\left\{3^{\frac{2}{3}}+\frac{2}{3} \int_{2}^{t}\left[1+2 e^{2 s}+4 s^{2}+8 \cos ^{2}(\pi s)\right] \Delta s\right\}^{\frac{3}{2}}, \quad t \in \mathbb{T}_{0} .
$$

\section{Competing interests}

The authors declare that they have no competing interests.

Authors' contributions

All authors contributed equally in this article. They read and approved the final manuscript.

\section{Author details}

${ }^{1}$ Nonlinear Dynamic Analysis Research Center, Department of Mathematics, Faculty of Applied Science, King Mongkut's University of Technology North Bangkok, Bangkok, 10800, Thailand. ${ }^{2}$ Department of Mathematics, University of loannina, loannina, 451 10, Greece. 


\section{Authors' information}

Sotiris K Ntouyas is a member of Nonlinear Analysis and Applied Mathematics (NAAM) Research Group at King Abdulaziz University, Jeddah, Saudi Arabia.

\section{Acknowledgements}

The research of P Thiramanus and J Tariboon is supported by King Mongkut's University of Technology North Bangkok, Thailand.

\section{Received: 26 November 2013 Accepted: 13 June 2014 Published: 22 July 2014}

\section{References}

1. Hilger, S: Analysis on measure chains - a unified approach to continuous and discrete calculus. Results Math. 18, 18-56 (1990)

2. Bohner, M, Peterson, A: Dynamic Equations on Time Scales: An Introduction with Applications. Birkhäuser, Basel (2001)

3. Feng, Q, Meng, F, Zhang, Y, Zhou, J, Zheng, B: Some delay integral inequalities on time scales and their applications in the theory of dynamic equations. Abstr. Appl. Anal. 2012, Article ID 538247 (2012)

4. Feng, Q, Meng, F, Zheng, B: Gronwall-Bellman type nonlinear delay integral inequalities on time scales. J. Math. Anal. Appl. 382, 772-784 (2011)

5. Li, W-N: Explicit bounds for some special integral inequalities on time scales. Results Math. 58, 317-328 (2010)

6. Li, W-N: Some delay integral inequalities on time scales. Comput. Math. Appl. 59, 1929-1936 (2010)

7. Li, W-N: Bounds for certain new integral inequalities on time scales. Adv. Differ. Equ. 2009, Article ID 484185 (2009)

8. Li, W-N, Sheng, W: Some nonlinear integral inequalities on time scales. J. Inequal. Appl. 2007, Article ID 70465 (2007)

9. Sun, Y: Some new integral inequalities on time scales. Math. Inequal. Appl. 15, 331-341 (2012)

10. Sun, Y: Some sublinear dynamic integral inequalities on time scales. J. Inequal. Appl. 2010, Article ID 983052 (2010)

11. Wang, $T, X u, R$ : Bounds for some new integral inequalities with delay on time scales. J. Math. Inequal. 6, 355-366 (2012)

12. Xu, R, Meng, F, Song, C: On some integral inequalities on time scales and their applications. J. Inequal. Appl. 2010, Article ID 464976 (2010)

13. Feng, Q, Zheng, B: Generalized Gronwall-Bellman-type delay dynamic inequalities on time scales and their applications. Appl. Math. Comput. 218, 7880-7892 (2012)

14. Feng, Q, Meng, F, Zhang, Y, Zheng, B, Zhou, J: Some nonlinear delay integral inequalities on time scales arising in the theory of dynamics equations. J. Inequal. Appl. 2011, Article ID 29 (2011)

15. Zheng, B, Feng, Q, Meng, F, Zhang, Y: Some new Gronwall-Bellman type nonlinear dynamic inequalities containing integration on infinite intervals on time scales. J. Inequal. Appl. 2012, Article ID 201 (2012)

16. Zheng, B, Zhang, Y, Feng, Q: Some new delay integral inequalities in two independent variables on time scales. J. Appl. Math. 2011, Article ID 659563 (2011)

17. Agarwal, R, Bohner, M, Peterson, A: Inequalities on time scales: a survey. Math. Inequal. Appl. 4, $535-557$ (2001)

18. Bainov, D, Hristova, S: Differential Equations with Maxima. Pure and Applied Mathematics. Chapman \& Hall/CRC, New York (2011)

19. Hristova, S, Stefanova, K: Some integral inequalities with maximum of the unknown functions. Adv. Dyn. Syst. Appl. 6 57-69 (2011)

20. Hristova, S, Stefanova, K: Linear integral inequalities involving maxima of the unknown scalar functions. J. Math Inequal. 4, 523-535 (2010)

21. Hristova, S, Stefanova, K: Nonlinear Bihari type integral inequalities with maxima. REMIA (2010)

22. Tariboon, J, Thiramanus, P, Ntouyas, SK: Dynamic integral inequalities on time scales with 'maxima'. J. Inequal. Appl. 2013, Article ID 564 (2013)

23. Jiang, F, Meng, F: Explicit bounds on some new nonlinear integral inequalities with delay. J. Comput. Appl. Math. 205 479-486 (2007)

24. Henderson, J, Hristova, S: Nonlinear integral inequalities involving maxima of unknown scalar functions. Math Comput. Model. 53, 871-882 (2011) 